\title{
Equações de intensidade-duração-frequência de chuvas para o estado do Piauí ${ }^{1}$
}

\author{
Intensity-duration-frequency equations for rainfall in the state of Piauí, Brazil
}

\author{
Alcinei Ribeiro Campos ${ }^{2 *}$, Glenio Guimarães Santos ${ }^{3}$, João Batista Lopes Silva ${ }^{3}$, João Irene Filho ${ }^{4}$ e Domingos de \\ Sousa Loura ${ }^{2}$
}

\begin{abstract}
RESUMO - Neste trabalho objetivou-se ajustar e comparar os parâmetros ( $K, a, b$ e $c)$ da equação de intensidadeduração-frequência para precipitações pluviais máximas de 133 estações pluviométricas localizadas no estado do Piauí, disponíveis no banco de dados da Agência Nacional de Águas - ANA. Inicialmente, foi realizada a seleção das estações através de analise de consistência das séries de dados, a partir do qual foram selecionadas 105 estações pluviométricas. As chuvas máximas de um dia foram desagregadas em duração de $5 ; 10 ; 15 ; 20 ; 25 ; 30 ; 60 ; 360 ; 480 ; 600 ; 720$ e 1.440 minutos e estimadas as chuvas máximas através de modelos probabilísticos para cada duração e período de retorno de 5 ; 10; 25; 50 e 100 anos. Em seguida, fez-se o ajuste dos parâmetros $(K, a, b$ e $c)$ através do emprego de regressão linear e não linear. O ajuste dos parâmetros da equação de intensidade-duração-frequência para precipitações máximas realizado por regressão não linear apresentou $R^{2}(0,99)$ superior aos valores ajustados por regressão linear $(0,98)$.
\end{abstract}

Palavras-chave: Hidrologia. Precipitação. Chuvas-Frequência da intensidade.

\begin{abstract}
This work aimed to adjust and compare the parameters (K, $a, b$ and $c)$ of the intensity-duration-frequency equations of the maximum rainfall taken from 133 rainfall stations located in the state of Piauí in Brazil, which are available from the database of the National Water Agency - ANA. The stations were initially chosen by analysis of the consistency of the data series, and then from these, 105 rainfall stations were then selected. The maximum rains for one day were separated into durations of $5,10,15,20,25,30,60,360,480,600,720$ and 1440 minutes and the maximum rainfall estimated using probabilistic models for each duration and each return period of 5, 10, 25, 50 and 100 years. The parameters $(K, a, b$ and $c)$ were then adjusted by means of both linear and nonlinear regression. The adjustment of the parameters of the intensity-duration- frequency equation for maximum precipitation, carried out using nonlinear regression, had $\mathrm{R}^{2}$ (0.99) higher than the values adjusted using linear regression (0.98).
\end{abstract}

Key words: Hydrology. Heavy rains. Return period.

\footnotetext{
*Autor para correspondência

${ }^{1}$ Recebido para publicação em 23/08/2013; aprovado em 26/03/2014

Pesquisa realizada em parceria com o programa de Pós-Graduação em Solos e Nutrição de Plantas da Universidade Federal do Piauí/PPGSN/UFPI ${ }^{2}$ Programa de Pós-Graduação em Solos e Nutrição de Plantas/PPGSN/UFPI, Campus Professora Cinobelina Elvas, BR 135, km três, Bom Jesus-PI, Brasil,64.900-000,jjalcinei@live.com,domingosloura@ hotmail.com

${ }^{3}$ Departamento de Engenharias, Universidade Federal do Piauí, Campus Professora Cinobelina Elvas, BR 135, km três, Bom Jesus-PI, Brasil, 64.900-000, gleniogm@gmail.com; silvajbl@yahoo.com.br

${ }^{4}$ Departamento de Medicina Veterinária, Universidade Federal do Piauí, Campus Professora Cinobelina Elvas, BR 135, km três, Bom Jesus-PI, Brasil, 64.900-000, joaoirenefilho@yahoo.com.br
} 


\section{INTRODUÇÃO}

Chuvas intensas, também chamadas de chuvas extremas, são aquelas que apresentam grandes lâminas precipitadas em pequenos intervalos de tempo (ARAÚJO et al., 2008; SILVA et al., 2003). Segundo Cecílio et al. (2009), por causar grandes escoamentos superficiais, as chuvas intensas são capazes de provocar prejuízos tanto em áreas urbanas quanto em áreas agrícolas, como inundação de terras cultivadas, erosão do solo, perdas de nutrientes e assoreamento de corpos da água, dentre outros. Dessa forma, a sua quantificação, bem como o conhecimento da forma como se distribui temporal e espacialmente são de extrema importância em estudos relacionados a dimensionamentos de projetos hidráulicos, como de irrigação, disponibilidade de água para abastecimento doméstico e industrial, obras de controle de inundação e erosão do solo (CECÍLIO et al., 2009; RODRIGUES et al., 2008; SANTOS; GRIEBELER; OLIVEIRA, 2010).

Nesse sentido, a quantificação dessas chuvas pode ser realizada por meio do emprego de equações de chuvas intensas, também denominadas de curvas intensidade-duração-frequência (IDF), que relacionam a duração, intensidade e frequência de ocorrência de um evento em determinado período de retorno (DAMÉ; TEIXEIRA; TERRA, 2008). O ajuste dos parâmetros das curvas IDF é realizado de modo empírico, a partir de dados pluviométricos para cada estação e local.

Já os parâmetros das curvas IDF podem ser ajustados por meio do emprego de regressão linear ou de regressão não linear (ARAGÃO et al., 2013; MELLO; SILVA, 2005; OLIVEIRA et al., 2005), com base em valores extraídos de séries de dados pluviométricos. Porém, o ajuste dos parâmetros da IDF apresenta algumas dificuldades, como exaustivo trabalho de tabulação, análise e interpretação de grande quantidade de dados de pluviogramas.

No Brasil, estudos pioneiros foram desenvolvidos por Pfafstetter (1957) e Denardin e Freitas (1982), em que foram ajustadas as equações IDF de 80 estações distribuídas por todo o país. Trabalhos mais recentes foram realizados por Oliveira et al. (2005), onde foram ajustadas as equações de IDF para Goiás e Distrito Federal, por Santos et al. (2009) para o Estado do Mato Grosso do Sul, e por Aragão et al. (2013) para o Estado de Sergipe.

No estado do Piauí, o trabalho pioneiro foi realizado por Denardin e Freitas (1982), em que foram ajustados os parâmetros da IDF apenas para a cidade de Teresina, sendo que apesar da extensão territorial e da variabilidade espacial das precipitações no Estado, este só dispõe dessa equação IDF. Assim, surge uma lacuna quando se faz necessário o emprego dessas equações para estimar eventos extremos utilizados no dimensionamento de obras hidráulicas no Estado, principalmente, obras voltadas para drenagem, manejo e conservação do solo e água.

Tendo em vista a grande importância do conhecimento das equações que relacionam intensidade-duração-frequência das precipitações e da inexistência dessas equações para a maioria dos municípios do Estado do Piauí, este trabalho teve como objetivo ajustar e comparar os parâmetros ( $K, a, b$ e $c$ ) da equação de intensidade-duração-frequência, por meio de regressão linear e não linear para 133 estações pluviométricas localizadas no Estado do Piauí.

\section{MATERIAL E MÉTODOS}

Para a realização deste trabalho foram utilizados dados pluviométricos coletados em 133 estações pluviométricas localizadas no estado da Piauí (Figura 1), disponíveis no banco de dados da Agência Nacional de Águas (ANA), em seu sítio eletrônico.

Para cada estação foram ajustados os parâmetros $K, a, b$, e $c$ da equação de intensidade, duração e frequência (Equação 1) por meio de regressão linear e não linear.

$$
\operatorname{Im}=\frac{K \cdot(T R)^{a}}{(t+b)^{c}}
$$

em que: Im - intensidade máxima média de precipitação, $\mathrm{mm} \mathrm{h}^{-1} ; T R$ - período de retorno, anos; $t$ - duração da precipitação, min.; $K, a, b$, e $c$ - parâmetros ajustados com base nos dados pluviométricos da localidade.

Inicialmente foi realizada uma análise de consistência das séries de dados de cada estação, sendo que para cada estação foram selecionados os anos que continham menor número de falhas nos dados, e descartadas as estações que apresentaram menos de 15 anos em sua série de dados, restando em 105 estações (Figura 1).

Após essa etapa, selecionou-se a precipitação máxima diária de cada ano das séries para cada estação analisada e, em seguida procedeu-se a desagregação das precipitações máximas de um dia para duração de $5 ; 10 ; 15 ; 20 ; 30 ; 60 ; 360 ; 480 ; 600 ; 720$ e 1.440 min., empregando-se os coeficientes propostos por Companhia de Tecnologia de Saneamento Ambiental (1986) (Tabela 1). Realizada a desagregação da precipitação fez-se a estimativa da precipitação máxima para cada período de retorno, e em seguida, o ajuste 
dos parâmetros $K, a, b$ e $c$ da equação de intensidade duração e frequência por dois métodos: (i) ajuste por regressão linear; e (ii) ajuste por regressão não linear.

Figura 1 - Distribuição espacial das estações selecionadas para ajuste das equações de IDF para o estado do Piauí

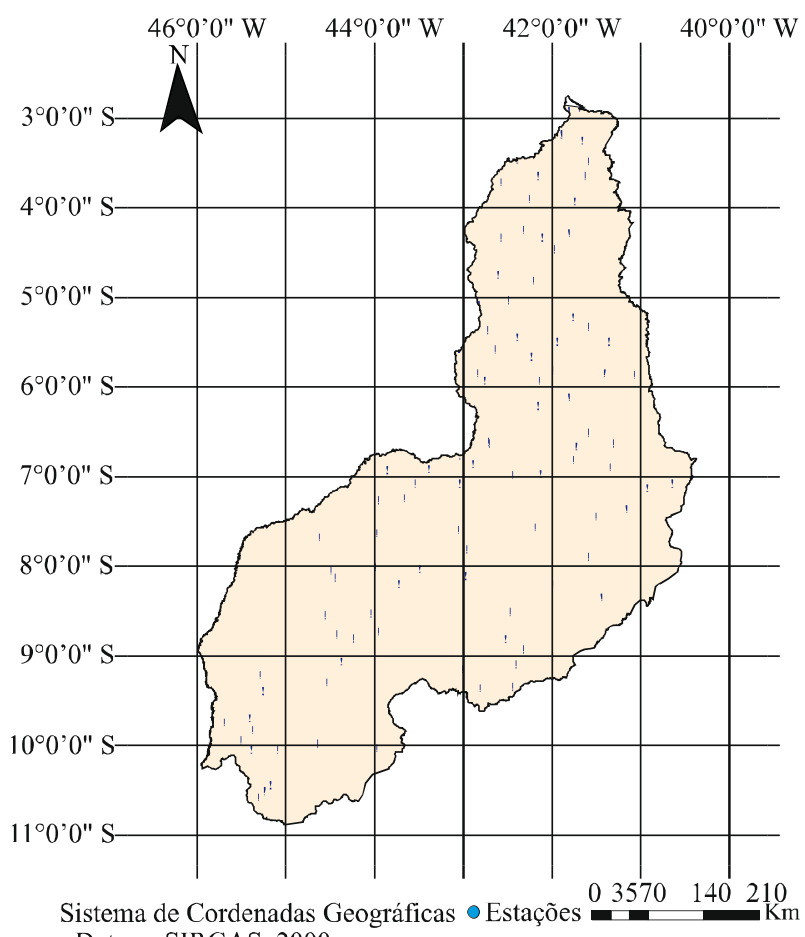

Datum: SIRGAS_2000

Tabela 1 - Coeficientes de desagregação para diferentes durações de chuva

\begin{tabular}{lc}
\hline Relação entre alturas pluviométricas & Fator de desagregação \\
\hline 1 dia/24 horas & 1,14 \\
12 horas/24 horas & 0,85 \\
10 horas/24 horas & 0,82 \\
8 horas/24 horas & 0,78 \\
6 horas/24 horas & 0,72 \\
1 hora/24 horas & 0,42 \\
30 min/1 hora & 0,74 \\
25 min/30 min & 0,91 \\
20 min/30 min & 0,81 \\
15 min/30 min & 0,70 \\
10 min/30 min & 0,54 \\
5 min/30 min & 0,34 \\
\hline
\end{tabular}

\section{Ajuste por regressão linear}

Depois de realizada a desagregação das chuvas em durações menores, foram estimados os eventos máximos para cada duração e períodos de retorno (TR) de 5; 10; 25; 50 e 100 anos, por meio do modelo probabilístico de Gumbel, conforme Equação 2.

$$
\mathrm{h}_{\mathrm{TR}}=\alpha-\beta \operatorname{Ln}\left[\operatorname{Ln}\left(\frac{\mathrm{TR}}{\mathrm{TR}-1}\right)\right]
$$

em que: $h_{T R}$ - altura pluviométrica associada a um período de retorno (TR) mm; $\alpha$ e $\beta$ - parâmetros da distribuição de Gumbel, obtidos pelo método dos momentos, Equações 3 e 4 .

$\mathrm{E}(\mathrm{h})=\alpha+0,577 \beta$

$\operatorname{VAR}(\mathrm{h})=1,645 \beta^{2}$

em que: $E(h)$ - valor esperado estimado pela média dos valores máximos anuais; $\operatorname{VAR}(h)$ - variância dos valores máximos anuais, estimada a partir da série de dados.

Após obtidos os valores das máximas precipitações para cada duração e período de retorno, realizou-se o ajuste dos parâmetros $(K, a, b$ e $c)$ da equação IDF (equação 1) para cada estação, pelo método dos mínimos quadrados. Para realizar o ajuste dos parâmetros empíricos da IDF, a Equação 1 foi reescrita da seguinte forma:

$\varphi=\mathrm{K} \cdot \mathrm{TR}^{\mathrm{a}}$

em que: $K$ e $a$ são parâmetros empíricos da equação IDF, e TR o período de retorno, em anos

$$
i=\frac{\varphi}{(t+b)^{c}}
$$

em que: $i=$ intensidade estimada; $t=$ tempo em minutos e $b$ e $c=$ parâmetros empíricos ajustados.

Em seguida, mediante a propriedade dos logaritmos a Equação 6 foi transformada na equação da reta, conforme Equação 7:

$\log \mathrm{i}=\log \varphi-\mathrm{c} \cdot \log (\mathrm{t}+\mathrm{b})$

$\mathrm{Na}$ sequência, foram calculados os logaritmos de intensidade máxima para cada duração (log i), em cada período de retorno. $\mathrm{O}$ valor de $\mathrm{b}$ foi obtido por iteração, utilizando-se a análise de regressão aplicada aos valores dos logaritmos da intensidade (log i) para um dado período de retorno, com relação aos logaritmos das durações $(\log$ t) (VILLELA; MATTOS, 1975).

A partir dos valores de $\log \mathrm{i}$ e $\log (\mathrm{t}+b)$ foram gerados gráficos de dispersão e, dessa forma, realizou-se 
o ajuste dos coeficientes angular (c) e linear $(\log \varphi)$, para cada período de retorno. Os parâmetros $K$ e $a$ da equação IDF foram estimados pelo método dos mínimos quadrados, conforme equação 8:

$\log \varphi=\log \mathrm{K}+\mathrm{a} \cdot \log \mathrm{TR}$

em que: $\log K$ e $a$ - coeficientes linear e angular da reta, respectivamente.

\section{Ajuste por regressão não linear}

Para cada estação, foram obtidas as séries máximas de alturas médias de precipitação de um dia, para os seguintes períodos de retorno (TR): $5 ; 10 ; 25 ; 50$ e 100 anos. As distribuições de probabilidade utilizadas foram: Gumbel; Log-Normal II; Log-Normal III; Pearson III; Log-Pearson III (TUCCI, 2001; NAGHETTINI; PINTO, 2007), sendo que para cada estação foram selecionadas as máximas precipitações, em que os dados da série apresentaram aderência ao modelo probabilístico pelo teste de Kolmogorov-Smirnov, e menor erro padrão. Todas estas etapas foram realizadas com auxílio do software SisCAH (SOUSA et al., 2009).

Em seguida, com os valores das séries máximas de alturas médias de precipitação de um dia, para as 105 estações para período de retorno de 5; 10; 25; 50 e 100 anos, fez-se a desagregação da precipitação de um dia em intervalos menores (Tabela 1). Depois de realizada a desagregação em tempos menores, foram ajustados, para cada estação, os parâmetros $K, a, b$, e $c$, por meio de regressão não linear, aplicando o método de interação de Gradação Reduzida Generalizada (GRG) Não Linear, através da rotina Solver da planilha eletrônica Excel ${ }^{\odot}$.

\section{Avaliação do ajuste realizado}

A avaliação do ajuste dos parâmetros da equação IDF, para ambas as metodologias (regressão linear e não linear) foi realizada pelo $\mathrm{R}^{2}$, obtida pelo quadrado da equação 9 .

$$
r=\frac{\sum(X-\bar{X}) \cdot(Y-\bar{y})}{\sqrt{\sum(X-\bar{X}) \cdot(y-\bar{y})}}
$$

em que: $\mathrm{X}$ - valores observados; $\overline{\mathrm{X}}$ - valores médios observados; $Y$ - valores estimados; $\overline{\mathrm{y}}$ - valores médios estimados.

Nesse mesmo sentido, fez-se a avaliação do ajuste dos dados pela equação de regressão dos valores observados, em relação aos valores estimados, analisando neste caso o coeficiente angular da reta; todas estas etapas realizadas empregando-se planilha eletrônica Excel ${ }^{\circ}$.

Ainda, foram realizadas as estimativas das intensidades máximas para duração de 10; 30; 60; 720 e 1.440 minutos, e período de retorno de $5 ; 10 ; 25 ; 50$ e 100 anos, para a cidade de Teresina. Em seguida, realizou-se a comparação com os valores estimados das duas metodologias com parâmetros ajustados por Denardin e Freitas (1982).

Após ajustados os parâmetros da IDF, por meio do emprego dos dois métodos descritos, foi realizada a espacialização dos parâmetros $K$ e $a$ para todo área territorial do Estado. A espacialização foi efetivada com auxilio do software ArcGIS ${ }^{\odot}$, versão 10, desenvolvido pela Environmental Systems Research Institute (ESRI), no qual foi utilizado o método de interpolação Kriging, do tipo ordinária (MELLO et al., 2003). Finalmente, foi realizada a espacialização das intensidades estimadas para duração de 30; 60 e 720 minutos para o período de retorno de 10 anos, também se utilizando o método de interpolação Kriging, do tipo ordinária.

\section{RESULTADOS E DISCUSSÃO}

O ajuste dos parâmetros $(K, a, b$ e $c)$ da IDF, tanto por regressão linear quanto os realizados por regressão não linear apresentam coeficientes de determinação superiores a 0,98 (Tabela 2). No ajuste dos parâmetros da IDF por regressão linear, apenas cinco das 105 estações estudadas, apresentam $r^{2}$ inferior a 0,99 . Já os parâmetros ajustados por meio de regressão não linear, todos apresentaram $\mathrm{R}^{2}$ superior a 0,99. Aragão et al. (2013) também obtiveram $\mathrm{r}^{2}$ acima de 0,99 para ajuste das IDF por regressão não linear, para o Estado de Sergipe, no entanto os autores alertam que esse índice pode ser tendencioso, podendo não ser adequado para avaliar o ajuste dos dados à equação de IDF.

No ajuste, por meio de regressão linear observase que há menor variação entre os parâmetros $K$ e $a$. O maior valor apresentado pelo parâmetro $K$ foi de 1.265,76 para a estação 00241000 (Luís Correia) e o menor valor foi 591,38 para a estação 00945003 (Monte Alegre do Piauí). O parâmetro $a$ variou de 0,2225 observado na estação 00842002 (São Raimundo Nonato), para 0,0985 observado na estação 00442008 (José de Freitas). Já os parâmetros $b$ e $c$ se mantiveram fixos para todas as estações, isto devido a metodologia de ajuste que fixa estes parâmetros para ajuste dos demais. Essa menor variação nos parâmetros $K$ e $a$, quando ajustados por regressão linear, provavelmente ocorrem em função da não variação nos parâmetros $b$ e $c$ da IDF.

Moruzzi e Oliveira (2009) sugeriram existir uma interação entre esses parâmetros $(K, a, b$ e $c)$, isto é, influência mútua entre as suas estimativas. O valor de um parâmetro é influenciado pelo valor do outro, entretanto, a combinação desses parâmetros geralmente resulta em bons modelos de previsão de IDF, fato observado nos parâmetros ajustados pelo método da regressão não linear. 
Tabela 2 - Parâmetros $(K, a, b$ e $c)$ da equação de intensidade-duração-frequência ajustados por regressão linear e por regressão não linear para 105 estações localizadas no estado do Piauí

\begin{tabular}{|c|c|c|c|c|c|c|c|c|c|c|c|c|}
\hline \multirow{2}{*}{$\begin{array}{l}\text { Código } \\
\text { estação }\end{array}$} & \multirow[t]{2}{*}{ da } & \multirow{2}{*}{ Município } & \multicolumn{5}{|c|}{ Paramentos ajustados por regressão linear } & \multicolumn{5}{|c|}{ Paramentos ajustados por regressão não linear } \\
\hline & & & K & $\mathrm{a}$ & $\mathrm{b}$ & $\mathrm{c}$ & $\mathrm{R}^{2}$ & K & $\mathrm{a}$ & $\mathrm{b}$ & $\mathrm{c}$ & $\mathrm{R}^{2}$ \\
\hline 00241000 & & Luís Correia & $1.265,76$ & 0,186 & 10 & 0,742 & 0,990 & $1.612,00$ & 0,223 & 14,39 & 0,791 & 0,998 \\
\hline 00241002 & & Parnaíba & $1.033,93$ & 0,151 & 10 & 0,742 & 0,994 & $1.281,70$ & 0,135 & 12,5 & 0,773 & 0,999 \\
\hline 00341000 & & Parnaíba & 763,77 & 0,195 & 10 & 0,742 & 0,991 & 798,54 & 0,214 & 10,9 & 0,757 & 0,999 \\
\hline 00341001 & & Buriti dos Lopes & 844,13 & 0,15 & 10 & 0,742 & 0,994 & $1.038,34$ & 0,141 & 12,68 & 0,775 & 0,999 \\
\hline 00341003 & & Cocal & 838,98 & 0,157 & 10 & 0,742 & 0,994 & 972,81 & 0,139 & 10,98 & 0,758 & 0,999 \\
\hline 00341004 & & Piracuruca & 658,88 & 0,168 & 10 & 0,742 & 0,993 & 976,26 & 0,118 & 13,22 & 0,78 & 0,999 \\
\hline 00341009 & & Piracuruca & 872,84 & 0,16 & 10 & 0,742 & 0,993 & 920,95 & 0,178 & 11,29 & 0,761 & 0,999 \\
\hline 00342002 & & Esperantina & 917,17 & 0,152 & 10 & 0,742 & 0,994 & $1.157,88$ & 0,139 & 12,89 & 0,777 & 0,999 \\
\hline 00342003 & & Esperantina & 862,92 & 0,134 & 10 & 0,742 & 0,995 & $1.063,01$ & 0,125 & 12,92 & 0,777 & 0,999 \\
\hline 00342004 & & Luzilândia & 832,82 & 0,139 & 10 & 0,742 & 0,995 & $1.021,92$ & 0,119 & 12,32 & 0,771 & 0,999 \\
\hline 00342005 & & Matias Olímpio & 939,00 & 0,115 & 10 & 0,742 & 0,996 & $1.083,21$ & 0,089 & 11,11 & 0,759 & 0,999 \\
\hline 00342006 & & Porto & $1.024,64$ & 0,151 & 10 & 0,742 & 0,994 & $1.010,13$ & 0,181 & 12,46 & 0,773 & 0,999 \\
\hline 00441002 & & Capitão de Campos & $1.033,56$ & 0,184 & 10 & 0,742 & 0,992 & $1.112,98$ & 0,205 & 12,36 & 0,772 & 0,999 \\
\hline 00441006 & & Piripiri & 898,00 & 0,145 & 10 & 0,742 & 0,995 & $1.045,17$ & 0,147 & 13,34 & 0,781 & 0,999 \\
\hline 00442000 & & Barras & $1.017,83$ & 0,14 & 10 & 0,742 & 0,995 & $1.131,01$ & 0,162 & 13,34 & 0,781 & 0,999 \\
\hline 00442001 & & Batalha & 995,84 & 0,123 & 10 & 0,742 & 0,994 & $1.130,24$ & 0,143 & 13,6 & 0,784 & 0,999 \\
\hline 00442004 & & Campo Maior & 962,94 & 0,126 & 10 & 0,742 & 0,994 & $1.075,28$ & 0,155 & 13,35 & 0,781 & 0,999 \\
\hline 00442006 & & Miguel Alves & 900,76 & 0,149 & 10 & 0,742 & 0,994 & $1.178,71$ & 0,13 & 12,46 & 0,773 & 0,999 \\
\hline 00442008 & & José de Freitas & $1.022,56$ & 0,099 & 10 & 0,742 & 0,995 & $1.126,62$ & 0,118 & 13 & 0,778 & 0,999 \\
\hline 00541001 & & São Miguel do Tapuio & 731,03 & 0,186 & 10 & 0,742 & 0,992 & 927,57 & 0,227 & 14,98 & 0,797 & 0,999 \\
\hline 00541002 & & Castelo do Piauí & 912,57 & 0,139 & 10 & 0,742 & 0,995 & $1.064,08$ & 0,135 & 12,62 & 0,774 & 0,999 \\
\hline 00541003 & & Castelo do Piauí & 851,19 & 0,158 & 10 & 0,742 & 0,994 & 744,36 & 0,224 & 11,61 & 0,764 & 0,996 \\
\hline 00541005 & & São Miguel do Tapuio & 776,32 & 0,147 & 10 & 0,742 & 0,994 & 968,80 & 0,116 & 12,32 & 0,771 & 0,999 \\
\hline 00541008 & & Aroazes & 920,46 & 0,152 & 10 & 0,742 & 0,994 & $1.084,05$ & 0,172 & 13,12 & 0,779 & 0,999 \\
\hline 00541009 & & São João da Serra & 957,53 & 0,165 & 10 & 0,742 & 0,993 & $1.218,75$ & 0,175 & 13 & 0,778 & 0,999 \\
\hline 00541010 & & São Miguel do Tapuio & 797,53 & 0,143 & 10 & 0,742 & 0,995 & $1.034,79$ & 0,129 & 12,91 & 0,777 & 0,999 \\
\hline 00542000 & & Altos & $1.029,32$ & 0,169 & 10 & 0,742 & 0,993 & $1.289,96$ & 0,178 & 13,27 & 0,78 & 0,998 \\
\hline 00542003 & & Beneditinos & $1.034,75$ & 0,155 & 10 & 0,742 & 0,994 & $1.319,95$ & 0,156 & 13,71 & 0,785 & 0,999 \\
\hline 00542004 & & Barro duro & $1.000,05$ & 0,151 & 10 & 0,742 & 0,994 & $1.128,55$ & 0,135 & 11,07 & 0,759 & 0,999 \\
\hline 00542005 & & Demerval Lobão & 886,81 & 0,137 & 10 & 0,742 & 0,995 & $1.142,60$ & 0,116 & 12,96 & 0,777 & 0,999 \\
\hline 00542007 & & Monsenhor Gil & 999,47 & 0,142 & 10 & 0,742 & 0,995 & $1.132,13$ & 0,134 & 11,49 & 0,763 & 0,999 \\
\hline 00542008 & & Prata do Piauí & 868,13 & 0,156 & 10 & 0,742 & 0,994 & 961,05 & 0,125 & 10,9 & 0,757 & 0,999 \\
\hline 00542009 & & São Félix do Piauí & $1.028,19$ & 0,168 & 10 & 0,742 & 0,993 & $1.226,05$ & 0,175 & 12,95 & 0,777 & 0,999 \\
\hline 00542010 & & São Pedro do Piauí & 910,97 & 0,149 & 10 & 0,742 & 0,994 & $1.209,20$ & 0,116 & 12,88 & 0,777 & 0,999 \\
\hline 00542012 & & Teresina & $1.055,61$ & 0,15 & 10 & 0,742 & 0,994 & $1.079,84$ & 0,202 & 12,76 & 0,776 & 0,999 \\
\hline 00543009 & & Palmeirais & 985,96 & 0,129 & 10 & 0,742 & 0,995 & $1.188,25$ & 0,14 & 12,43 & 0,772 & 0,999 \\
\hline 00641000 & & Aroazes & 936,11 & 0,151 & 10 & 0,742 & 0,994 & $1.137,68$ & 0,163 & 12,55 & 0,773 & 0,999 \\
\hline 00641001 & & Bocaina & 757,91 & 0,167 & 10 & 0,742 & 0,993 & 970,71 & 0,179 & 13,29 & 0,781 & 0,999 \\
\hline 00641002 & & Pimenteiras & 835,57 & 0,169 & 10 & 0,742 & 0,993 & $1.077,60$ & 0,182 & 13,36 & 0,781 & 0,999 \\
\hline 00641003 & & Inhuma & 811,51 & 0,191 & 10 & 0,742 & 0,991 & $1.044,92$ & 0,205 & 13,35 & 0,781 & 0,999 \\
\hline 00641004 & & Ipiranga do Piauí & 743,15 & 0,145 & 10 & 0,742 & 0,994 & 917,95 & 0,157 & 12,77 & 0,776 & 0,999 \\
\hline 00641005 & & Valença do Piauí & 800,06 & 0,157 & 10 & 0,742 & 0,994 & $1.014,14$ & 0,171 & 13,1 & 0,779 & 0,999 \\
\hline
\end{tabular}


Continuação Tabela 2

\begin{tabular}{|c|c|c|c|c|c|c|c|c|c|c|c|}
\hline 00642000 & Oeiras & 927,79 & 0,19 & 10 & 0,742 & 0,991 & $1.178,62$ & 0,206 & 13,09 & 0,779 & 0,999 \\
\hline 00642001 & Amarante & 952,82 & 0,149 & 10 & 0,742 & 0,994 & $1.182,64$ & 0,16 & 12,85 & 0,776 & 0,999 \\
\hline 00642003 & Amarante & 898,11 & 0,14 & 10 & 0,742 & 0,995 & $1.146,92$ & 0,156 & 13,18 & 0,78 & 0,999 \\
\hline 00642004 & Floriano & 848,00 & 0,16 & 10 & 0,742 & 0,994 & $1.086,26$ & 0,176 & 13,22 & 0,78 & 0,999 \\
\hline 00642005 & Elesbão Veloso & $1.018,94$ & 0,21 & 10 & 0,742 & 0,990 & $1.446,08$ & 0,226 & 14,67 & 0,794 & 0,998 \\
\hline 00642007 & Amarante & 818,24 & 0,178 & 10 & 0,742 & 0,992 & $1.280,71$ & 0,191 & 16,25 & 0,808 & 0,998 \\
\hline 00642012 & Oeiras & 840,24 & 0,173 & 10 & 0,742 & 0,993 & $1.112,01$ & 0,192 & 13,63 & 0,784 & 0,999 \\
\hline 00643002 & Jerumenha & 761,45 & 0,175 & 10 & 0,742 & 0,992 & $1.000,44$ & 0,191 & 13,6 & 0,784 & 0,999 \\
\hline 00643006 & Floriano & 680,07 & 0,174 & 10 & 0,742 & 0,993 & 885,81 & 0,188 & 13,51 & 0,783 & 0,999 \\
\hline 00643008 & Guadalupe & 692,92 & 0,209 & 10 & 0,742 & 0,990 & 979,42 & 0,225 & 14,62 & 0,793 & 0,998 \\
\hline 00740001 & São Julião & 803,43 & 0,148 & 10 & 0,742 & 0,994 & 997,61 & 0,16 & 12,84 & 0,776 & 0,999 \\
\hline 00740002 & Fronteiras & 759,05 & 0,161 & 10 & 0,742 & 0,993 & 963,14 & 0,173 & 13,15 & 0,779 & 0,999 \\
\hline 00741001 & Conceição do Canindé & 902,87 & 0,163 & 10 & 0,742 & 0,993 & $1.151,45$ & 0,176 & 13,21 & 0,78 & 0,999 \\
\hline 00741003 & Itainópolis & 785,74 & 0,169 & 10 & 0,742 & 0,993 & $1.011,10$ & 0,181 & 13,35 & 0,781 & 0,999 \\
\hline 00741004 & Jaicós & 775,12 & 0,163 & 10 & 0,742 & 0,993 & 996,13 & 0,182 & 13,63 & 0,784 & 0,999 \\
\hline 00742000 & Flores do Piauí & 927,86 & 0,191 & 10 & 0,742 & 0,991 & $1.246,18$ & 0,204 & 13,97 & 0,787 & 0,999 \\
\hline 00742001 & Simplício Mendes & 608,53 & 0,2 & 10 & 0,742 & 0,991 & 854,99 & 0,215 & 14,56 & 0,793 & 0,998 \\
\hline 00742012 & Francisco Ayres & 758,61 & 0,132 & 10 & 0,742 & 0,995 & 933,12 & 0,15 & 12,63 & 0,774 & 0,999 \\
\hline 00743000 & Bertolinia & 860,81 & 0,149 & 10 & 0,742 & 0,994 & $1.042,04$ & 0,161 & 12,49 & 0,773 & 0,999 \\
\hline 00743001 & Itaueira & 957,85 & 0,153 & 10 & 0,742 & 0,994 & $1.208,20$ & 0,168 & 13,02 & 0,778 & 0,999 \\
\hline 00743002 & Itaueira & 860,06 & 0,161 & 10 & 0,742 & 0,993 & $1.091,19$ & 0,173 & 13,15 & 0,779 & 0,999 \\
\hline 00743003 & Jerumenha & 914,18 & 0,145 & 10 & 0,742 & 0,994 & $1.133,45$ & 0,159 & 12,8 & 0,776 & 0,999 \\
\hline 00743004 & Landri Sales & $1.128,30$ & 0,165 & 10 & 0,742 & 0,993 & $1.441,76$ & 0,177 & 13,25 & 0,78 & 0,999 \\
\hline 00743009 & Jerumenha & 894,23 & 0,106 & 10 & 0,742 & 0,997 & $1.046,56$ & 0,129 & 12,22 & 0,77 & 0,999 \\
\hline 00744001 & Uruçuí & 869,89 & 0,159 & 10 & 0,742 & 0,994 & $1.104,49$ & 0,173 & 13,14 & 0,779 & 0,999 \\
\hline 00841001 & Conceição do Canindé & 651,05 & 0,166 & 10 & 0,742 & 0,993 & 840,18 & 0,181 & 13,34 & 0,781 & 0,999 \\
\hline 00841002 & São João do Piauí & 724,34 & 0,168 & 10 & 0,742 & 0,993 & 936,85 & 0,183 & 13,38 & 0,782 & 0,999 \\
\hline 00842000 & São João do Piauí & 828,45 & 0,16 & 10 & 0,742 & 0,993 & $1.033,35$ & 0,175 & 12,87 & 0,777 & 0,999 \\
\hline 00842001 & Canto do Buriti & 889,92 & 0,169 & 10 & 0,742 & 0,993 & $1.123,33$ & 0,183 & 13,06 & 0,778 & 0,999 \\
\hline 00842002 & São Raimundo Nonato & 826,06 & 0,223 & 10 & 0,742 & 0,989 & $1.084,65$ & 0,239 & 13,53 & 0,783 & 0,998 \\
\hline 00842003 & São Raimundo Nonato & 781,22 & 0,172 & 10 & 0,742 & 0,993 & $1.026,96$ & 0,189 & 13,56 & 0,783 & 0,999 \\
\hline 00843001 & Eliseu Martins & 895,58 & 0,195 & 10 & 0,742 & 0,991 & $1.232,03$ & 0,212 & 14,22 & 0,789 & 0,999 \\
\hline 00843002 & Eliseu Martins & 790,59 & 0,172 & 10 & 0,742 & 0,993 & $1.023,28$ & 0,185 & 13,43 & 0,782 & 0,999 \\
\hline 00843004 & Cristino Castro & 682,30 & 0,157 & 10 & 0,742 & 0,994 & 944,65 & 0,174 & 14,32 & 0,79 & 0,999 \\
\hline 00843007 & Bertolínia & 804,75 & 0,172 & 10 & 0,742 & 0,993 & 1047,92 & 0,186 & 13,48 & 0,783 & 0,999 \\
\hline 00844000 & Uruçuí & 867,12 & 0,178 & 10 & 0,742 & 0,992 & $1.141,09$ & 0,193 & 13,64 & 0,784 & 0,999 \\
\hline 00844001 & Uruçuí & 847,48 & 0,153 & 10 & 0,742 & 0,994 & $1.062,68$ & 0,166 & 12,97 & 0,778 & 0,999 \\
\hline 00844002 & Bom Jesus & 739,12 & 0,146 & 10 & 0,742 & 0,994 & 918,16 & 0,168 & 13,01 & 0,778 & 0,999 \\
\hline 00844004 & Bertolínia & 784,02 & 0,168 & 10 & 0,742 & 0,993 & $1.014,76$ & 0,183 & 13,39 & 0,782 & 0,999 \\
\hline 00844005 & Cristino Castro & 657,47 & 0,195 & 10 & 0,742 & 0,991 & 901,31 & 0,211 & 14,19 & 0,789 & 0,999 \\
\hline 00844006 & Bertolínia & 873,89 & 0,186 & 10 & 0,742 & 0,992 & $1.172,44$ & 0,202 & 13,9 & 0,787 & 0,999 \\
\hline 00844008 & Cristino Castro & 801,19 & 0,176 & 10 & 0,742 & 0,992 & $1.047,83$ & 0,19 & 13,57 & 0,783 & 0,999 \\
\hline 00941000 & São Raimundo Nonato & 818,74 & 0,159 & 10 & 0,742 & 0,994 & $1.038,91$ & 0,173 & 13,13 & 0,779 & 0,999 \\
\hline 00942000 & Caracol & 812,65 & 0,142 & 10 & 0,742 & 0,995 & 999,17 & 0,154 & 12,71 & 0,775 & 0,999 \\
\hline 00942001 & São Raimundo Nonato & 886,73 & 0,17 & 10 & 0,742 & 0,993 & $1.157,49$ & 0,187 & 13,48 & 0,783 & 0,999 \\
\hline
\end{tabular}


Continuação Tabela 2

\begin{tabular}{|c|c|c|c|c|c|c|c|c|c|c|c|}
\hline 00942002 & São Raimundo Nonato & 792,01 & 0,168 & 10 & 0,742 & 0,993 & $1.023,87$ & 0,182 & 13,38 & 0,782 & 0,999 \\
\hline 00942003 & São Raimundo Nonato & 767,19 & 0,138 & 10 & 0,742 & 0,995 & $1.019,51$ & 0,15 & 13,8 & 0,786 & 0,999 \\
\hline 00944000 & Bom Jesus & 724,58 & 0,165 & 10 & 0,742 & 0,993 & 945,02 & 0,189 & 13,89 & 0,786 & 0,999 \\
\hline 00944001 & Bom Jesus & 812,93 & 0,142 & 10 & 0,742 & 0,995 & $1.004,38$ & 0,156 & 12,75 & 0,775 & 0,999 \\
\hline 00944002 & Parnaguá & 783,85 & 0,166 & 10 & 0,742 & 0,993 & $1.011,41$ & 0,181 & 13,34 & 0,781 & 0,999 \\
\hline 00945001 & Barreiras do Piauí & 889,13 & 0,164 & 10 & 0,742 & 0,993 & $1.139,61$ & 0,178 & 13,27 & 0,78 & 0,999 \\
\hline 00945002 & Gilbues & 718,37 & 0,143 & 10 & 0,742 & 0,995 & 889,31 & 0,157 & 12,77 & 0,776 & 0,999 \\
\hline 00945003 & Monte Alegre do Piauí & 591,38 & 0,192 & 10 & 0,742 & 0,991 & 808,26 & 0,208 & 14,15 & 0,789 & 0,999 \\
\hline 00945005 & Gilbués & 874,20 & 0,136 & 10 & 0,742 & 0,995 & $1.071,49$ & 0,154 & 12,71 & 0,775 & 0,999 \\
\hline 00945007 & Gilbués & 685,49 & 0,154 & 10 & 0,742 & 0,994 & 870,12 & 0,188 & 13,52 & 0,783 & 0,999 \\
\hline 00945008 & Gilbués & 931,32 & 0,164 & 10 & 0,742 & 0,993 & $1.057,55$ & 0,179 & 11,51 & 0,763 & 0,999 \\
\hline 01043000 & Avelino Lopes & 677,04 & 0,194 & 10 & 0,742 & 0,991 & 914,71 & 0,207 & 14,05 & 0,788 & 0,999 \\
\hline 01044004 & Curimatá & 796,94 & 0,133 & 10 & 0,742 & 0,995 & 976,97 & 0,149 & 12,61 & 0,774 & 0,999 \\
\hline 01045000 & Corrente & 762,12 & 0,15 & 10 & 0,742 & 0,994 & 960,07 & 0,167 & 12,98 & 0,778 & 0,999 \\
\hline 01045001 & Parnaguá & 888,30 & 0,142 & 10 & 0,742 & 0,995 & $1.008,32$ & 0,205 & 12,73 & 0,775 & 0,999 \\
\hline 01045002 & Parnaguá & 862,43 & 0,154 & 10 & 0,742 & 0,994 & $1.081,99$ & 0,167 & 12,99 & 0,778 & 0,999 \\
\hline 01045003 & Parnaguá & 596,20 & 0,222 & 10 & 0,742 & 0,989 & 866,40 & 0,238 & 15,06 & 0,797 & 0,998 \\
\hline 01045004 & Curimatá & 854,86 & 0,139 & 10 & 0,742 & 0,995 & $1.073,19$ & 0,161 & 12,86 & 0,777 & 0,999 \\
\hline
\end{tabular}

Nos parâmetros ajustados por regressão não linear observa-se que há grande variação entre seus valores, o que pode ser explicado pela interação entres esses parâmetros, conforme relatado por Moruzzi e Oliveira (2009). O maior valor apresentado para o parâmetro $K$ foi de 1.612,0 para a estação 00241000 (Luís Correia) e o menor valor $(744,3634)$ para a estação 00541003 (Castelo do Piauí). O parâmetro $a$ apresentou variação de 0,0888 para a estação 00342005 (Matias Olímpio) e 0,2392 para a estação 00842002 (São Raimundo Nonato). Já o parâmetro $b$ variou de 16,246 para a estação 00642007 (Amarante) e 10,8959 para a estação 00341000 (Parnaíba).

Nesse mesmo sentido, o parâmetro $c$ apresentou variação de 0,0512 entre o valor máximo e o mínimo. Esses resultados são semelhantes aos encontrados por Silva et al. (2003) e Silva et al. (2012), quando realizaram o ajuste dos parâmetros da equação de IDF empregando regressão não linear. No entanto, diverge dos resultados encontrados por Aragão et al. (2013), no qual os parâmetros $b$ e $c$ se mantiveram fixos quando ajustados por regressão não linear.

Essa grande variação nos parâmetros da equação IDF, dentro de uma mesma faixa territorial também foram observadas por Silva et al. (2002), Rodrigues et al. (2008), Santos et al. (2009) e Silva et al. (2012). Dessa forma, variações podem ocorrer em função das características e da variabilidade dos eventos hidrológicos de cada Região. Portanto, os resultados apresentados evidenciam a importância de se realizar o ajuste dos parâmetros da IDF para cada local específico.

Na espacialização do parâmetro $K$, observase que os maiores valores ajustados, tanto por regressão linear (Figura 2A) quanto por regressão não linear (Figura 2B) estão distribuídos de forma mais concentrados na região Norte e Centro-Norte piauiense. Isso ocorre em função dessas localidades apresentarem as maiores intensidades de precipitação pluviométrica.

Já o paramento $a$ tem seus maiores valores observados nas regiões Sudoeste e Sudeste do Estado (Figura 3). Nesse sentido, o parâmetro $\boldsymbol{a}$ ajustado por regressão linear (Figura 3A) e por regressão não linear (Figura 3B) apresentou comportamento contrário ao parâmetro $K$, sendo que o parâmetro $a$ apresenta seus menores valores nas regiões Norte e Centro-Norte piauiense. A distribuição espacial dos parâmetros $K$ e $a$ evidenciam sua forte ligação com os valores de intensidade de precipitação que ocorrem em uma determinada região, sendo que as regiões que apresentaram os maiores valores de intensidade foram também aquelas onde se observou maiores valores para o parâmetro $K$ e valores menores para o parâmetro $a$.

De acordo com os valores de intensidade máxima estimados com as IDF ajustadas por regressão linear (Tabela 3), pode se observar que as estações 
que apresentaram os maiores e menores valores de intensidades máximas estimadas para todas as durações foram: a estação 00241000 localizada no município de Luís Correia, e a estação 00945003 localizada no município de Monte Alegre do Piauí, respectivamente.
Esse comportamento pode ser explicado pela distribuição espacial das chuvas no Estado, onde os maiores valores de pluviosidade ocorrem na região Norte e os menores na região Central, região Semiárida e no Sul do Estado (GUEDES et al., 2010).

Figura 2 - Espacialização do parâmetro K ajustado por regressão linear (A) e regressão não linear (B) respectivamente
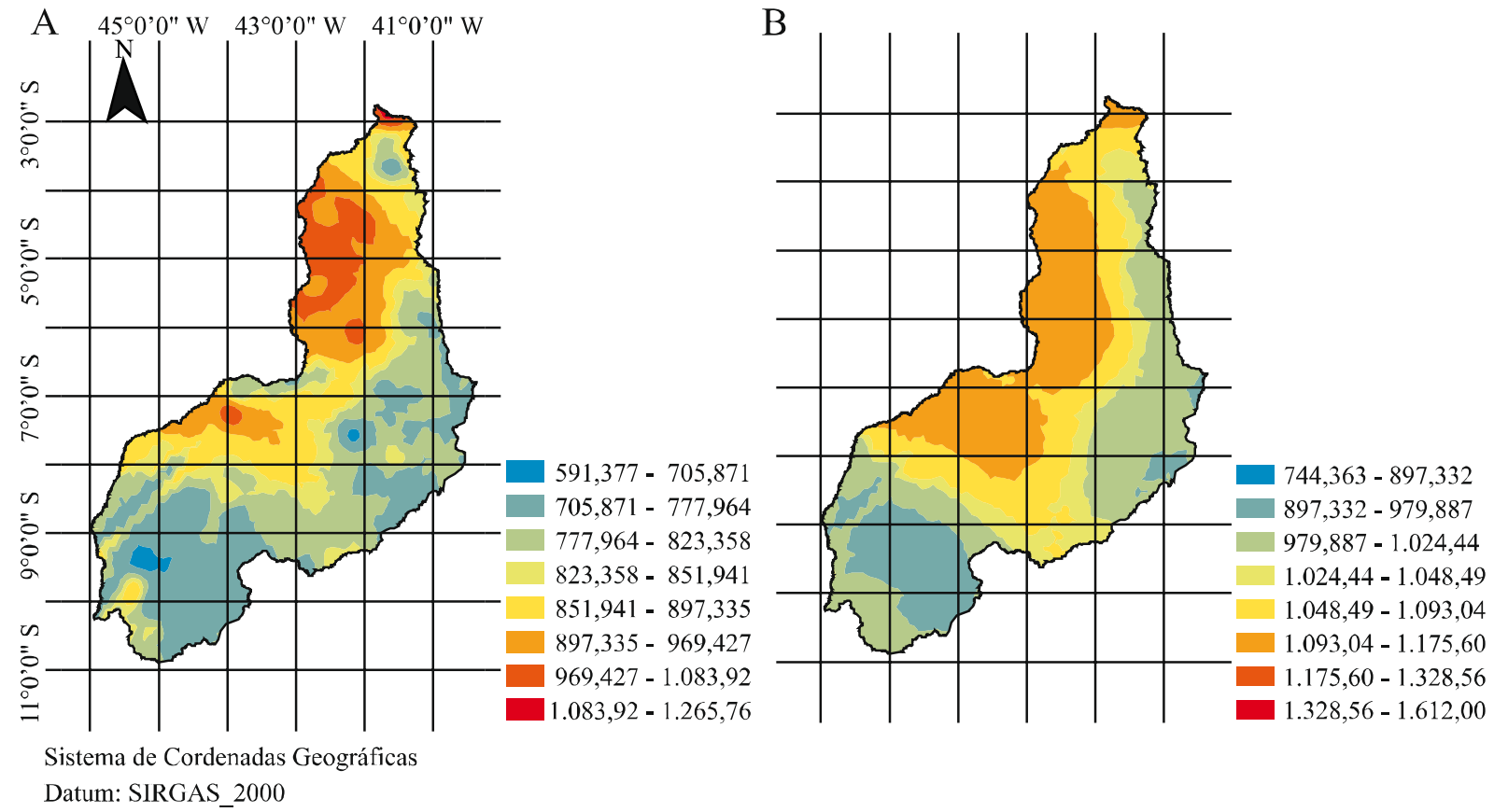

Figura 3 - Espacialização do parâmetro a ajustado por regressão linear (A) e regressão não linear (B) respectivamente
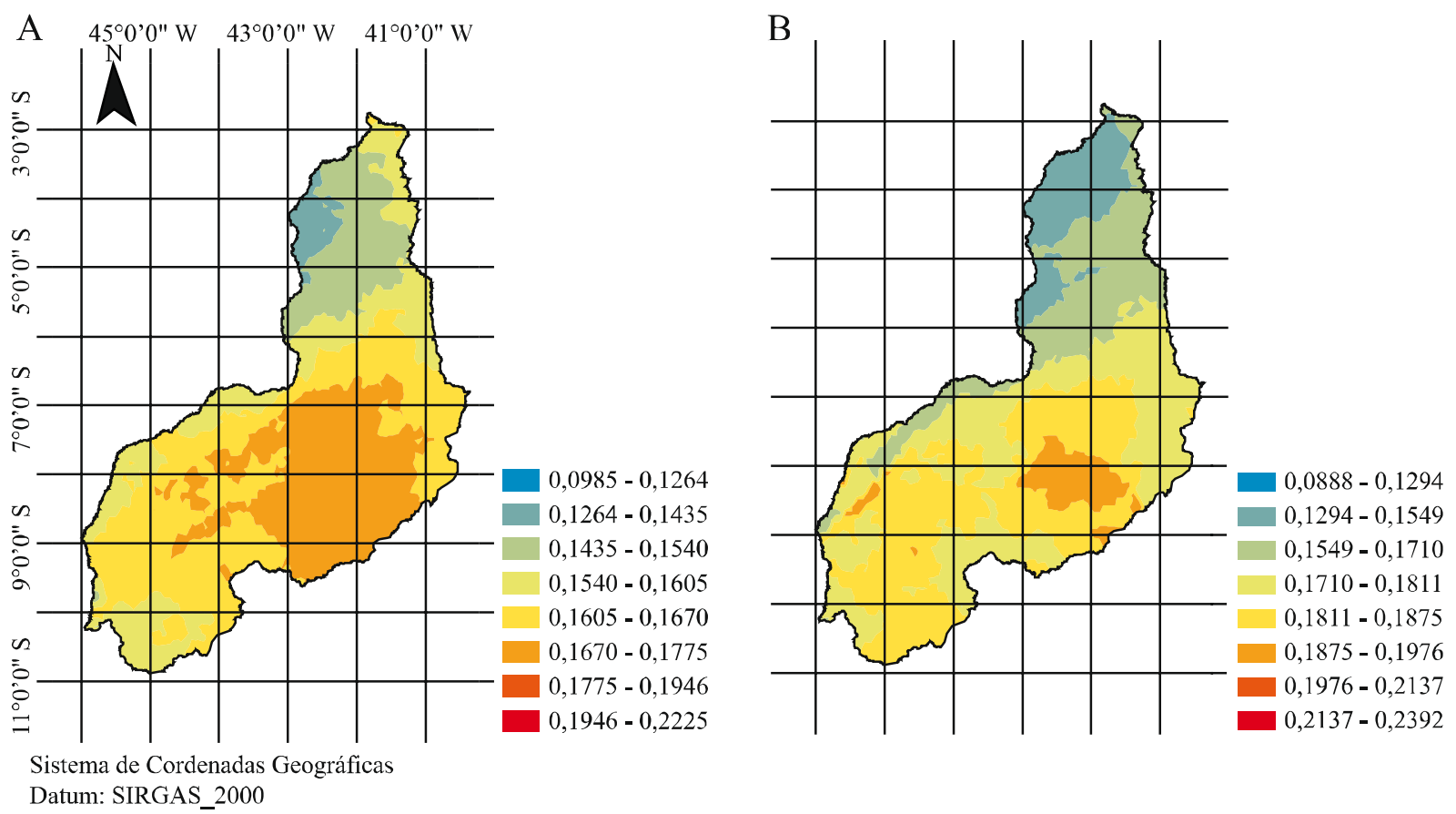
Tabela 3 - Intensidades máximas estimadas com parâmetros ajustados por regressão linear e por regressão não linear para duração de $10,30,60,720$ e 1440 minutos e período de retorno de 10 anos

\begin{tabular}{|c|c|c|c|c|c|c|c|c|c|c|c|}
\hline \multirow{2}{*}{$\begin{array}{l}\text { Código da } \\
\text { estação }\end{array}$} & \multirow{2}{*}{ Município } & \multicolumn{5}{|c|}{$\begin{array}{c}\text { Precipitação estimada com paramentos ajustados } \\
\text { por regressão linear }\end{array}$} & \multicolumn{5}{|c|}{$\begin{array}{c}\text { Precipitação estimada com paramentos ajustados por } \\
\text { regressão não linear }\end{array}$} \\
\hline & & $10 \mathrm{~min}$ & $30 \mathrm{~min}$ & $60 \mathrm{~min}$ & $720 \mathrm{~min}$ & $\begin{array}{r}1440 \\
\min \end{array}$ & $10 \mathrm{~min}$ & $30 \mathrm{~min}$ & $60 \mathrm{~min}$ & $720 \mathrm{~min}$ & $1440 \mathrm{~min}$ \\
\hline 00241000 & Luís Correia & 210,39 & 125,80 & 83,06 & 14,59 & 8,77 & 215,33 & 134,09 & 89,13 & 14,57 & 8,48 \\
\hline 00342006 & Porto & 157,09 & 93,93 & 62,01 & 10,89 & 6,55 & 138,54 & 84,70 & 56,05 & 9,38 & 5,53 \\
\hline 00642005 & Elesbão Veloso & 178,86 & 106,95 & 70,61 & 12,40 & 7,45 & 191,19 & 119,34 & 79,38 & 12,93 & 7,52 \\
\hline 00945003 & Monte Alegre do Piauí & 99,64 & 59,58 & 39,34 & 6,91 & 4,15 & 105,68 & 65,67 & 43,62 & 7,15 & 4,17 \\
\hline
\end{tabular}

Quando se utilizou os parâmetros ajustados por regressão linear para estimar a intensidade máxima na duração de 10 min., a estação 00241000 (Luís Correia) apresentou valor de $210,39 \mathrm{~mm} \mathrm{~h}^{-1} \mathrm{e}$ a estação 00945003 (Monte Alegre do Piauí) apresentou valor de $99,64 \mathrm{~mm} \mathrm{~h}^{-1}$. Já quando se utilizou os parâmetros ajustados por regressão não linear para estimar a intensidade máxima na duração de 10 min., a estação 00241000 (Luís Correia) apresentou valor de 215,33 $\mathrm{mm} \mathrm{h}^{-1}$ e a estação 00945003 (Monte Alegre do Piauí) apresentou valor de 105,68 $\mathrm{mm} \mathrm{h}^{-1}$. Entretanto, quando se observa a variação entre os valores estimados com duração de 10 min. para uma mesma estação utilizando os parâmetros ajustados, tanto por regressão linear quanto por regressão não linear, nota-se que os valores apresentaram pequena variação, sendo que para a estação 00241000 (Luís Correia), a variação foi de apenas $4,94 \mathrm{~mm} \mathrm{~h}^{-1}$, e para a estação 00945003 (Monte Alegre do Piauí) foi de $6,04 \mathrm{~mm} \mathrm{~h}^{-1}$. Essa diferença pode ser justificada pela diferença existente entre as metodologias.

Ao se observar os valores de intensidade máxima estimados com os parâmetros ajustados por regressão linear e por regressão não linear, verificase que a estação 00342006 (Porto) apresentou maiores variações para duração de 10; 720 e 1.440 min., apresentando variação de 18,$55 ; 1,55$ e $1,02 \mathrm{~mm} \mathrm{~h}^{-1}$, respectivamente. Já para a duração de 30 e 60 min., a estação 00642005 (Elesbão Veloso) apresentou as maiores diferenças, com valores da ordem de 12,39 e $8,77 \mathrm{~mm} \mathrm{~h}^{-1}$, na sequência apresentada.

Por outro lado, a espacialização das intensidades máximas estimadas para período de retorno de 10 anos e duração de 10; 60 e 720 min., com os parâmetros da IDF ajustados por regressão linear (Figuras 4A, 4B e 4C) e regressão não linear (Figuras 4D, 4E e 4F) apresentam comportamento similar de distribuição espacial no Estado.

No entanto, as intensidades estimadas com os parâmetros ajustados por regressão linear apresentaram maiores áreas com maiores e menores valores de precipitação que a regressão não linear, principalmente ao Norte, onde ocorrem as maiores precipitações, e ao Sul, onde ocorrem as menores precipitações. Pode se observar ainda que as maiores intensidades de precipitação ocorrem principalmente, na região Norte e Centro-Norte piauiense, e os menores valores nas regiões Sudoeste e Sudeste do Estado. Esses resultados confirmam a distribuição dos parâmetros $K$ e $a$ no Estado, sendo que nas regiões que apresentam maiores valores de $K$ e menores valores de $a$, tendem a apresentar maiores valores na intensidade precipitada.

De acordo com a Figura 5, pode-se observar que existe uma semelhança entre os valores de intensidade estimados utilizando-se os parâmetros ajustados neste trabalho e os valores estimados com os parâmetros ajustados por Denardin e Freitas (1982). Essa semelhança é comprovada por meio da correlação entre os valores de intensidade de chuva, com $\mathrm{R}^{2}$ superior a 0,98 . A comparação entre os valores estimados com os parâmetros ajustados por meio de regressão linear (IRL), com os valores estimados com os parâmetros ajustados por Denardin e Freitas (1982) propiciaram $\mathrm{R}^{2}$ de 0,98. Já a comparação entre os valores estimados com os parâmetros ajustados, por intermédio de regressão não linear (IRNL), com os valores de intensidades estimadas com os parâmetros ajustados por Denardin e Freitas (1982) (IDFe) exibiram $\mathrm{R}^{2}$ superior a 0,99 . 
Figura 4 - Espacialização das intensidades máximas estimadas para período de retorno de 10 anos com os parâmetros da IDF ajustados por regressão linear e duração de $10 \mathrm{~min}$ (A), $60 \mathrm{~min}$ (B) e $720 \mathrm{~min}$ (C) e valores estimados com parâmetros ajustados por regressão não linear com duração de $10 \min (\mathrm{D}), 60 \mathrm{~min}(\mathrm{E}) 720 \mathrm{~min}(\mathrm{~F})$

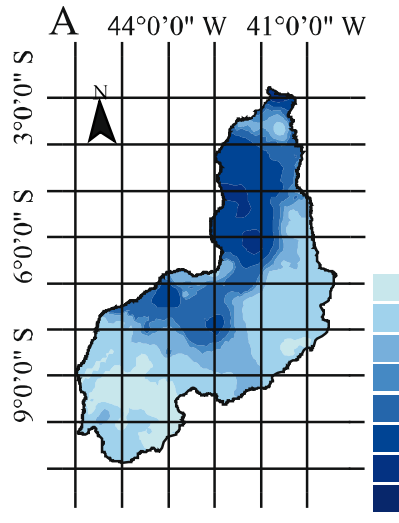

$\mathrm{D}$

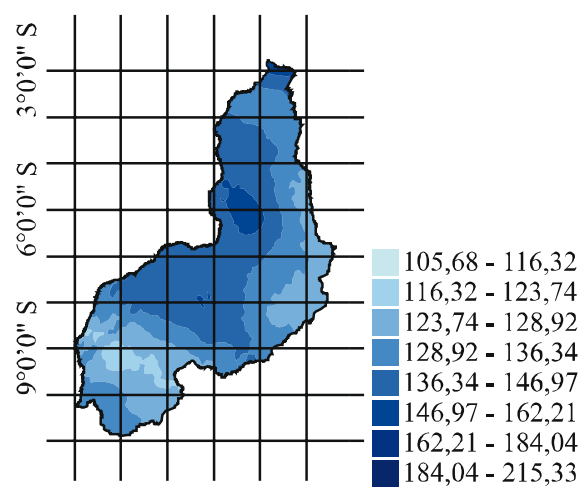

Sistema de Cordenadas Geográficas

Datum: SIRGAS_2000
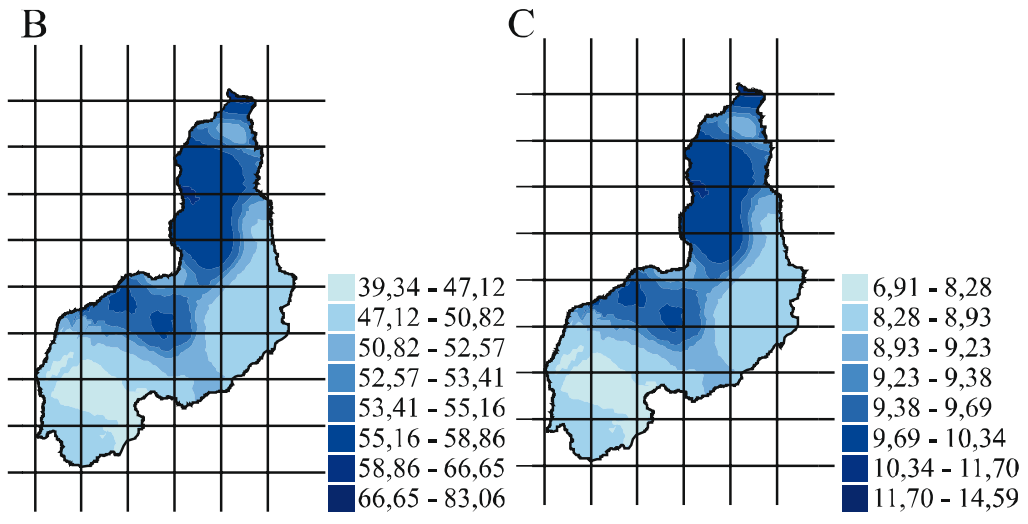

$\mathrm{E}$

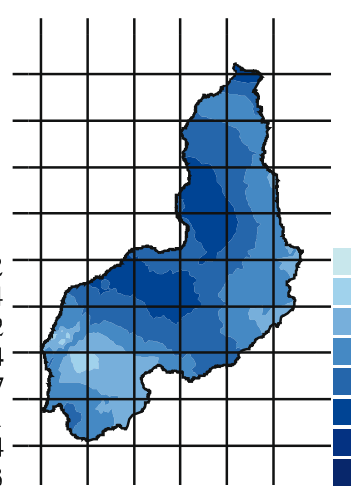

$\mathrm{F}$

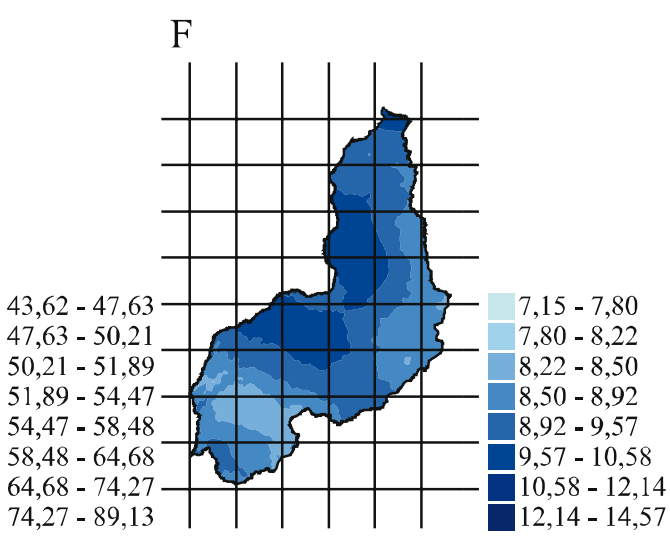

$74,27-89,13$
Figura 5 - Comparação entres valores estimados com parâmetros ajustados por regressão linear (IRL) e por regressão não linear (IRNL) em relação aos valores estimados com parâmetros ajustados por Denardin e Freitas (1982) (IDFe) para a cidade de Teresina

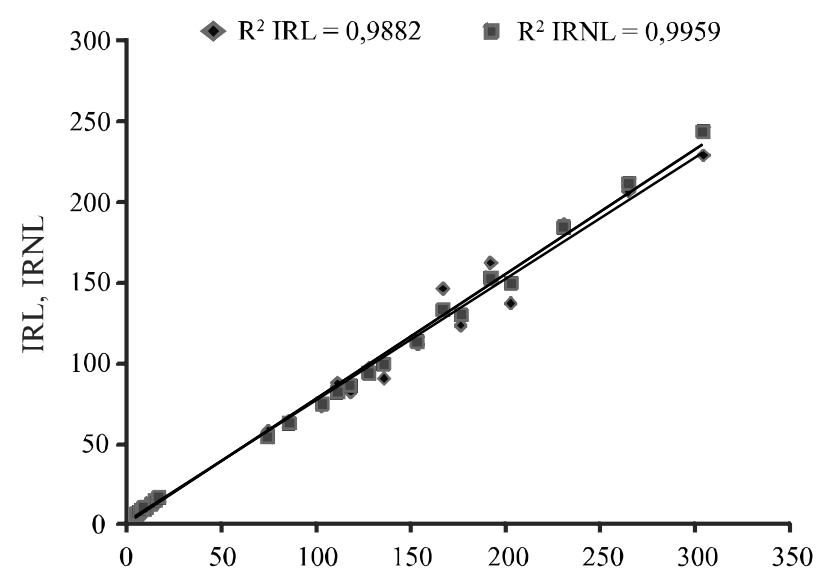

\section{CONCLUSÕES}

1.O ajuste dos parâmetros das equações de intensidadeduração-frequência realizado, tanto por regressão linear quanto por regressão não linear foram bons, com $\mathrm{r}^{2}$ superior a 0,98 ;

2.O método de regressão não linear apresentou melhor desempenho quando comparado com o método de regressão linear;

3. A espacialização do parâmetro $K$ mostrou que os maiores valores ocorrem nas regiões Norte e CentroNorte piauiense, enquanto para o parâmetro a observa-se comportamento contrário;

4.A comparação das intensidades estimadas com os parâmetros ajustados neste trabalho, com os valores estimados com os parâmetros disponíveis para o município de Teresina apresentam $\mathrm{R}^{2}$ superior a 0,98 , evidenciando o bom ajuste da equação. 


\section{REFERÊNCIAS}

AGÊNCIA NACIONAL DE ÁGUAS. Hidroweb - Sistemas de Informações Hidrológicas. 2012. Disponível em: <http:// hidroweb.ana.gov.br>. Acesso em: 28 de março 2013.

ARAGÃO, R. et al. Chuvas intensas para o estado de Sergipe com base em dados desagregados de chuva diária. Revista Brasileira de Engenharia Agrícola e Ambiental, v. 17, n. 3, p. 243-252, 2013.

ARAÚJO, L. E. et al. Análise estatística de chuvas intensas da bacia hidrográfica do Rio Paraíba. Revista Brasileira de Meteorologia, v. 23, n. 2, p. 162-169, 2008.

CECÍlIO, R. A. et al. Avaliação de interpoladores para os parâmetros das equações de chuvas intensa no Espirito Santo. Ambi-Água, v. 4, n. 3, p. 82-92, 2009.

COMPANHIA DE TECNOLOGIA DE SANEAMENTO AMBIENTAL. Drenagem urbana: manual de projeto. São Paulo: DAEE/CETESB, 1986. 466 p.

DAMÉ, R. C. F.; TEIXEIRA, C. F. A; TERRA, V. S. S. Comparação de diferentes metodologias para estimativa de curvas intensidade-duração-frequência para pelotas - RS. Revista Engenharia Agrícola, v. 28, n. 2, p. 245-255, 2008.

DENARDIN, J.; FREITAS, P. L. Características fundamentais da chuva no Brasil. Pesquisa Agropecuária Brasileira, v. 17, n. 10, p. 1409-1416, 1982.

GUEDES, R. V. S. et al. C. Análise em componentes principais da precipitação pluvial no estado do Piauí e agrupamento pelo método de ward. Revista de Geografia, v. 27, n. 1, p. 208-233, 2010.

MELLO, C. R. et al. Krigagem e inverso do quadrado da distância para interpolação dos parâmetros da equação de chuvas intensas. Revista Brasileira de Ciência do Solo, v. 27, n. 5 , p. $925-933,2003$.

MELLO, C. R.; SILVA, A. M. Métodos estimadores dos parâmetros da distribuição de Gumbel e sua influência em estudos hidrológicos de projeto. Irriga, v. 10, n. 4, p. 318334, 2005.

MORUZZI, R. B.; OLIVEIRA, S. C. Relação entre intensidade, duração e frequência de chuvas em Rio Claro,
SP: métodos e aplicação. Teoria e Prática da Engenharia Civil, v. 9, n. 13, p. 59-68, 2009.

NAGHETTINI, M.; PINTO, E. J. A. Hidrologia Estatística. Belo Horizonte: CPRM, 2007. 552 p.

OLIVEIRA, L. F. C. et al. Intensidade-duração-frequência de chuvas intensas para localidades no Estado de Goiás e Distrito Federal. Pesquisa Agropecuária Tropical, v. 35, n. 1, p. 13-18, 2005.

PFAFstetTer, O. Chuvas intensas no Brasil. Brasília: Departamento Nacional de Obras e Saneamento, 1957. 246 p.

RODRIGUES, J. O. et al. Equações de intensidade-duraçãofrequência de chuvas para as localidades de Fortaleza e Pentecoste, Ceará. Scientia Agraria, v. 9, n. 4, p. 511-519, 2008.

SANTOS, G. G. et al. Intensidade-duração-frequência de chuvas para o Estado de Mato Grosso do Sul. Revista Brasileira de Engenharia Agrícola e Ambiental, v. 13, p. $899-905,2009$. Suplemento.

SANTOS, G. G.; GRIEBELER, N. P.; OLIVEIRA, L. F. C. Chuvas intensas relacionadas à erosão hídrica. Revista Brasileira de Engenharia Agrícola e Ambiental, v. 14, n. 2, p. 115-123, 2010.

SILVA, B. M. et al. Chuvas Intensas em localidades do Estado de Pernambuco. Revista Brasileira de Recursos Hídricos, v. 17, n. 3, p. 135-147, 2012.

SILVA, D. D. et al. Equações de intensidade-duraçãofrequência da precipitação pluvial para o Estado de Tocantins. Engenharia na Agricultura, v. 11, n. 4, p. 1-8, 2003.

SILVA, D. D. et al. Chuvas intensas no Estado da Bahia. Revista Brasileira de Engenharia Agrícola e Ambiental, v. 6, n. 2, p. 362-367, 2002.

SOUSA, H. T. et al. SisCAH - Sistema Computacional para Análise Hidrológica. Versão 1.0. GPRH, 2009.

TUCCI, C. E. M. Hidrologia: ciência e aplicação. 2. ed. Porto Alegre: UFRGS, 2001. 943 p.

VILlELA, S. M.; MATTOS, A. Hidrologia Aplicada. São Paulo: McGraw Hill do Brasil, 1975. 245 p. 